\title{
UM-IU@LING at SemEval-2019 Task 6: Identifying Offensive Tweets Using BERT and SVMs
}

\author{
Jian Zhu \\ Department of Linguistics \\ University of Michigan \\ Ann Arbor, MI, USA \\ lingjzhu@umich.edu
}

\author{
Zuoyu Tian \\ Department of Linguistics \\ Indiana University \\ Bloomington, IN, USA \\ zuoytianeiu.edu
}

\author{
Sandra Kübler \\ Department of Linguistics \\ Indiana University \\ Bloomington, IN, USA \\ skuebler@indiana.edu
}

\begin{abstract}
This paper describes the UM-IU@LING's system for the SemEval 2019 Task 6: OffensEval. We take a mixed approach to identify and categorize hate speech in social media. In subtask A, we fine-tuned a BERT based classifier to detect abusive content in tweets, achieving a macro $F_{1}$ score of 0.8136 on the test data, thus reaching the 3rd rank out of 103 submissions. In subtasks B and C, we used a linear SVM with selected character $n$-gram features. For subtask C, our system could identify the target of abuse with a macro $F_{1}$ score of 0.5243 , ranking it 27 th out of 65 submissions.
\end{abstract}

\section{Introduction}

With the increased influence of social media on modern society, large amounts of user-generated content emerge on the internet. Besides the exchange of ideas, we also see an exponential increase of aggressive and potentially harmful content, for example, hate speech. If we consider the amount of user-generated data, it is impractical to manually identify the malicious speech. Thus we need to develop methods to detect offensive speech automatically through computational models. However, this task is challenging because natural language is fraught with ambiguities, and language in social media is extremely noisy. Here we present our method to automatically identifying offensive content in tweets.

We primarily focus on detecting whether a tweet contains offensive content or not (subtask A), and then determining the target of the offensive content (subtask C). For subtask A, we use pre-trained word embeddings by fine-tuning the BERT model (Devlin et al., 2018) for detecting offensive tweets. For subtasks B and C, BERT did not perform well, either because of limited training data or because we did not find the appropriate hyperparameters. Thus we use an SVM classifier with character $n$-grams as features. We accidentally flipped the predicted labels in our submission to subtask B, which is why we do not report results of subtask B here. Among all teams participating in OffensEval, our models ranks 3rd out of 103 on subtask A and 27th out of 65 on subtask C. (see Zampieri et al., 2019b).

\section{Related Work}

Detecting offensive language online is becoming more and more important (Schmidt and Wiegand, 2017; Founta et al., 2018; Malmasi and Zampieri, 2018). To build an effective classifier, one of the major problems is to find the appropriate features. Normally, two types of features are utilized: surface features like $n$-grams and word representations trained by neural network. Most offensive language classifiers are trained on different types of surface features with approaches like SVM (Malmasi and Zampieri, 2018; Arroyo-Fernández et al., 2018), Random Forest (Burnap and Williams, 2015), and Logistic Regression (Davidson et al., 2017). Recently, word embeddings trained in neural networks have been shown to achieve good performance in offensive language identification tasks (Badjatiya et al., 2017). Benchmarks of the first shared task on aggression identification (Kumar et al., 2018) show that half of the top 15 systems are trained on neural networks.

Using pre-trained word embeddings for feature extraction has been shown to be highly effective in multiple NLP tasks. Traditional word embeddings are extracted from shallow neural networks trained on a large swathes of texts required to learn the contextual representations of words. Examples include skip-grams (Mikolov et al., 2013) and GloVe (Pennington et al., 2014). However, these embeddings are learned from an aggregation of all possi- 
ble word contexts, which may gloss over semantic nuances in representations.

Recent models like ELMo (Peters et al., 2018) and BERT (Devlin et al., 2018) significantly advanced the state-of-the-art in language modeling by learning context-sensitive representations of words. ELMo goes beyond word embeddings by learning representations that are functions of the entire input sentence (Peters et al., 2018). However, ELMo is still considered shallow with two bidirectional LSTM layers, and more recent transformer based language models such as the OpenAI Generative Pre-trained Transformer (GPT) (Radford et al., 2018) and Bidirectional Encoder Representations from Transformers (BERT) (Devlin et al., 2018) have been extended to a depth of up to twelve layers. The OpenAI GPT is still a unidirectional language model while BERT is trained to be bidirectional with two novel prediction tasks, Masked LM and Next Sentence Prediction. The pre-trained BERT model has been shown to give significant improvements in a series of downstream tasks over ELMo and OpenAI GPT (Devlin et al., 2018).

However, identifying offensive language is not a simple task. Challenges during identification include but are not limited to the fact that surface language features fail to capturing subtle semantic difference, and the shortage of undisputed annotated data (Malmasi and Zampieri, 2018). Most of previous studies focus on distinguishing between offensive and non-offensive language (Kwok and Wang, 2013; Djuric et al., 2015), which is the goal of subtask A in the current shared task. But part of challenge consists of the intertwined nature of such messages having negative connotations and profanity. Dinakar et al. (2011) show that it is important to tease these two factors apart. Malmasi and Zampieri (2018) first address the issue of distinguishing hate speech from general profanity.

\section{Methodology and Data}

The subtasks in the shared task are rather different. In subtask A, the goal is to identify offensive tweets; in subtask B and C, the aim is to distinguish targeted and untargeted offense and to classify the targeted ones into different types. Subtask A requires sensitivity to subtle changes in word meaning in context while the other subtasks are more categorical in nature. However, both suffer from data sparsity. Therefore, we decided, backed by empirical validation on the trial data, to utilize different methods for the subtasks, namely, BERT embeddings for subtask A, and an SVM classifier for subtasks B and C.

The data collection method used to compile the dataset in OffensEval is described by Zampieri et al. (2019a). We used the official training data and trial data provided by the shared task to train the classifier. Our implmentations can be found at: https://github.com/zytian9/SemEval-2019-Task-6.

\subsection{Subtask A: Identifying Abusive Content}

The goal of subtask A is to identify whether a tweet contains offensive content by training a model to perform binary classification. There are 13,240 tweet instances in the training data, in which each instance has been labeled as either 'offensive' ('OFF') or 'not offensive' ('NOT'). The model takes a tweet as input and predicts the corresponding label of that tweet. We used the trial data as development data.

\subsubsection{Model Details}

For subtask A, we trained a classifier by finetuning a pre-trained BERT Transformer (Devlin et al., 2018) with a linear layer for text sequence classification on top.

The input sentences ${ }^{1}$ were first tokenized with the BERT basic tokenizer to perform punctuation splitting, lower casing and invalid characters removal. Then this was followed by WordPiece tokenization (Wu et al., 2016) to split words into subword units, in accordance with the original BERT approach (Devlin et al., 2018). The maximum sequence length was defined as 80 , with shorter sequences padded and longer sequences truncated to this length. The order of the input sequence was represented by the learned positional embeddings. The input representation for each tweet is the sum of these token, segment, and position embeddings. As only one sentence serves as input, only the sentence A embeddings are used as the segment embeddings (Devlin et al., 2018).

We selected the BERT $_{\text {base-uncased }}$ as the underlying BERT model. The BERT base $_{\text {consists of }}$ 12 Transformer blocks, 12 self-attention heads, and 768 hidden dimension with a total parameters of $110 \mathrm{M}$. It was trained on the BookCorpus ( $800 \mathrm{M}$ words) and the English Wikipedia (2,500M

\footnotetext{
${ }^{1}$ In BERT, a "sentence" can be a text sequence of arbitrary length. In our case, a "sentence" refers to a tweet even if it may span multiple linguistic sentences.
} 
words). Though the $\mathrm{BERT}_{\text {large }}$ model was reported to outperform the BERT $_{\text {base }}$ in a variety of tasks, training and fine tuning BERT $_{\text {large }}$ was too computationally intensive given the time limit. Thus we used $\mathrm{BERT}_{\text {base }}$ for accelerated training. The $\mathrm{BERT}_{\text {base }}$ model includes a special classification embedding [CLS ] at the beginning of every sentence, and this token in the final layer was extracted as the aggregate sequence representation for the current classification task. Then a linear layer of 768 dimensions was added on top of $\mathrm{BERT}_{\text {base}}$, using the [CLS] embeddings of the whole input sequence to predict a binary label. Binary cross-entropy was used as the loss function to fine-tune the classifier.

\subsubsection{Implementation}

The neural network was implemented in PyTorch (Paszke et al., 2017), and we used the tokenizer, pretrained WordPiece, and positional embeddings and pre-trained BERT from the library pytorch-pretrained-bert ${ }^{2}$. Following the recommendation for fine-tuning in the original BERT approach (Devlin et al., 2018), we trained our classifier with a batch size of 32 for 2 epochs. The dropout probability was set to 0.1 for all layers. Adam optimizer was used with a learning rate of 2e-5. The training was carried out on an Nvidia 1070Ti GPU; only taking about 6 minutes in total.

\subsection{Subtask B: Categorizing Offense Types}

For subtasks B and C, we adopted an SVM classifier. For these two tasks, the BERT classifier performed close to the baseline on the trial data. This could be caused by the limited amount of the training data for these two tasks or inappropriate selection of hyperparameters. Thus, we built a linear SVM classifier to identify the offense type and target.

Subtask B requires the distinction between targeted and untargeted offense. We used an SVM classifier with selected character $n$ gram features for subtask B. For the trial data of subtask B, the classifier achieved a macro $F_{1}$ score of 0.5333 and accuracy of 0.5714 ; both of them considerably higher than the baseline. But since the labels of two classes were accidentally flipped in our submission, our results were not competitive. We also reconstructed test $F_{1}$ from the flipped confusion

\footnotetext{
BERT
}

matrix. If the labels were not flipped, the test $\mathrm{F}_{1}$ should be 0.5946 .

\subsection{Subtask C: Identifying the Target of Abuse}

Subtask $\mathrm{C}$ requires the classifier to identify three types of offense target, 'Individual' ('IND'), 'Group'('GRP') and 'Other'('OTH'). The training set is rather imbalanced: The minority class OTH constitutes around 10 percent of all the instances, and only occurs once in the trial data. We originally were planning to use the same approach as for subtasks A. However, experiments on the trial data showed a weak performance. For this reason, we decided to use a linear SVM classifier to identify the offense target with three sub-classes since previous studies indicate that SVM classifiers perform well on classification tasks and at par with deep neural networks when features are well selected (Founta et al., 2018; Kumar et al., 2018). For this classifier, we used the Scikit-learn (Pedregosa et al., 2011) implementation, and we used only the training data provided by the shared task.

\subsubsection{Model Details}

Given that character-level $n$-gram could reduce the effect of spelling errors and variations in tweets (Schmidt and Wiegand, 2017), we used a bag of character $n$-grams (with $n$ ranging from 2 to 7 characters) as features in order to characterize the users' language features as robustly as possible. Since in subtask C, we need to identify different types of offense target, we assume that named entity information will be effective for identifying target types. Named entities information was extracted by spaCy, which is based on the entity types from OntoNotes 5 corpus $^{3}$. Given that this task aims to identify three types of targets, namely individual, group and other, we used the named entity information by classifying all the entity types into three major types and counting the number of each type separately. The first type only includes PERSON entities, the second type consists of entity types related to a group sense, for example ORG, NORG, and GPE, and the last type includes all occurrences of the other entity types.

Nobata et al. (2016) found that linguistic features such as tweet length, average word length,

\footnotetext{
${ }^{3}$ https: / / spacy.io/api/annotation\# named-entities
} 


\begin{tabular}{l|rr} 
System & F $_{1}$ macro & Accuracy \\
\hline All OFF baseline & 0.2182 & 0.2790 \\
All NOT baseline & 0.4189 & 0.7209 \\
\hline BERT $_{\text {base-uncased }}$ & $\mathbf{0 . 8 1 3 6}$ & 0.8570
\end{tabular}

Table 1: The official UM-IU@LING result for subtask $\mathrm{A}$, in comparison to the baselines.

\begin{tabular}{l|rr} 
System & F $_{1}$ macro & Accuracy \\
\hline All OFF baseline & 0.1934 & 0.2399 \\
All NOT baseline & 0.4319 & 0.7601 \\
\hline SVM $_{\text {character-ngram }}$ & 0.8267 & 0.8782 \\
BERT $_{\text {base-uncased }}$ & $\mathbf{0 . 8 3 8 8}$ & 0.8722 \\
BERT $_{\text {base-cased }}$ & 0.8094 & 0.8500 \\
BERT $_{\text {base-multiling-unc. }}$ & 0.4300 & 0.7625 \\
BERT $_{\text {base-multiling-cased }}$ & 0.8179 & 0.8718 \\
\hline
\end{tabular}

Table 2: Results on the trial data for subtask A.

number of punctuation, number of discourse connectives can be useful for detecting abusive language. In this study, we adopt 9 features from their work. Besides the $n$-gram features, named entity, and linguistic features, we also adopted emoji and emoticons as additional features, which have been shown to be useful in sentiment analysis tasks (Kouloumpis et al., 2011; Shiha and Ayvaz, 2017). Emoticons are extracted using the s regular expressions by C. Potts ${ }^{4}$. We also added three emoji sentiment features, which consist of the positive, negative, and overall sentiment scores based on the Emoji Sentiment Ranking (Novak et al., 2015).

We performed feature selection for the $n$-gram features using a filtering approach with information gain, which has proven to be effective in social media sentiment classification (Kübler et al., 2018).

Our final submission is a linear SVM classifier $(\mathrm{C}=0.1$, squared-hinge loss function) with 1000 selected character $n$-grams of length 2-7. Adding linguistic and emoji features resulted in small gains on the trial data and was that not considered useful for the official version.

\section{Results}

\subsection{Subtask A}

Our best result for subtask A along with the official baselines are summarized in Table 1. The

\footnotetext{
${ }^{4}$ http://sentiment.christopherpotts.net/tokenizing.html \#emoticons
}

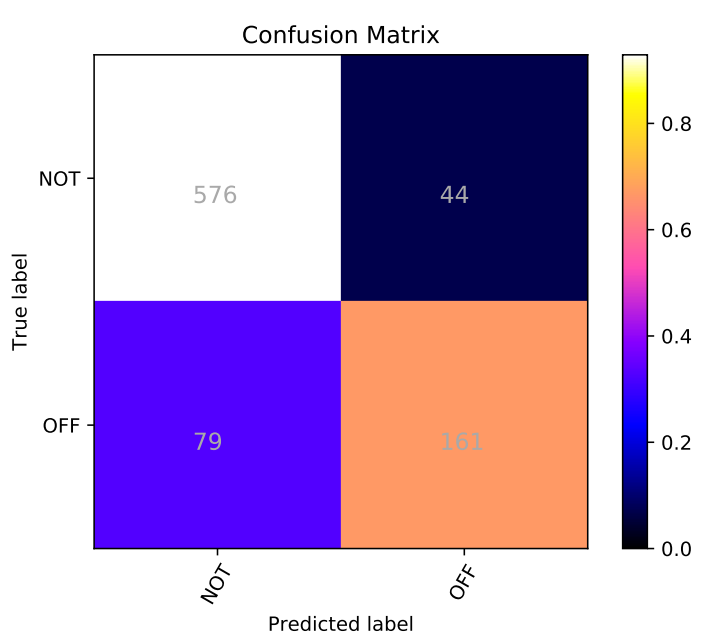

Figure 1: The UM-IU@LING confusion matrix for subtask A.

BERT classifier achieved a macro $F_{1}$ score of 0.8136 , clearly exceeding the baseline of 0.4189 and ranking the system 3rd out of 103 submissions. This demonstrates that our model can effectively identify whether a given tweet contains offensive content or not. The confusion matrix in Figure 1 further illustrates the error pattern of our classifier, which more often misclassified offensive tweets as being not offensive. One explanation of the results may be the classifier's preference for the majority class. But it is possible that our classifier may not capture some of the subtle nuances in meaning and contexts. However, the results also show that the macro $F_{1}$ score is only about 4.5 percent points lower than the accuracy ( 0.8136 vs. 0.8570$)$. This is a clear indication that the classifier is successful in modeling the minority class of offensive tweets.

\subsubsection{Ablation Analysis}

We performed an ablation analysis on our BERT classifier using the training and the trial data. First, we retrained the classifier by varying the learning rate. The macro $F_{1}$ dropped to the baseline of 0.4318 with a learning rate of either $2 \mathrm{e}-8$ or $2 \mathrm{e}-$ 3 , which indicates that the system is sensitive to change in learning rates.

The selection of sequence length only has a minimal influence on the final performance, with a tendency for longer sequence length to improves prediction accuracy: Setting the input sequence length to 60 reduces the macro $F_{1}$ minimally to 0.8212 , and decreasing the input length to $40 \mathrm{de}-$ creases the macro $F_{1}$ to 0.8126 . 


\begin{tabular}{l|l|l|l} 
ID & Tweet & Label & Prediction \\
\hline 50 & $\begin{array}{l}\text { okay but it actually sucks so much that the first year I COULD go to } \\
\text { every Reeperbahn Festival day, I'm in Strasbourg and can only attend } \\
\text { the last day }\end{array}$ & NOT & OFF \\
\hline 263 & $\begin{array}{l}\text { My mom just called me and said she is joining the NFL boycott. How } \\
\text { many of yall are with us? F that league \#NFLBoycott }\end{array}$ & OFF & NOT \\
\hline 126 & $\begin{array}{l}\text { @User @User @ User They don't. The GOP will keep supporting rack- } \\
\text { eteer, illegitimate Trump. They never will stop the corruption of tRump. } \\
\text { They are in it for the money. They want to destroy American democracy. }\end{array}$ & NOT & OFF \\
\hline
\end{tabular}

Table 3: Misclassified examples for subtask A from the trial data. Usernames are anonymized.

There are several versions of pre-trained BERT $_{\text {base }}{ }^{5}$. We compared the performance of these different versions of BERT $\mathrm{Base}_{\text {and }}$ ane results are summarized in Table 2. Generally, these variants of BERT $_{\text {base }}$ tend to give similar perfor-

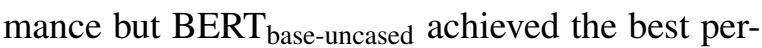
formance on the trial data. It is unclear why $\mathrm{BERT}_{\text {base-multilingual-uncased }}$ did not learn to perform the task beyond the baseline. Additional hyperparameter tuning might be necessary in this case. Overall, these results demonstrate that though BERT can give superior performance in detecting hate speech, it is somewhat sensitive to the change of hyperparameters. We also find that the SVM classifier achieved a higher accuracy on the trial data, but there is a significant drop in macro $\mathrm{F}_{1}$ when compared with the BERT model. This shows that the BERT model performs better on the minority class.

\subsubsection{Error Analysis}

We show examples of misclassified tweets in Table 3. In example 263, the BERT classifier failed to identify the offensive word "F". It is common for people to use euphemisms to tone down swear words in certain situations. The classifier could miss these word variants, especially when the word variant is the only offensive word in the given tweet. For tweet 50, the word "sucks" is the only word that is often used offensively. However, the given tweet is not offensive because the author only describes their mood instead of insulting someone else. These misclassifications seem to indicate that the classifier reacts to trigger words with negative connotations but may not be capable of interpreting the words with respect to the larger context.

When examining the prediction errors, we con-

\footnotetext{
${ }^{5}$ https://github.com/google-research/bert
}

\begin{tabular}{l|rr} 
System & F $_{1}$ macro & Accuracy \\
\hline All GRP baseline & 0.1787 & 0.3662 \\
All IND baseline & 0.2130 & 0.4695 \\
All OTH baseline & 0.0941 & 0.1643 \\
\hline SVM classifier & $\mathbf{0 . 5 2 4 3}$ & 0.6854 \\
\hline
\end{tabular}

Table 4: The official UM-IU@LING results (SVM) for subtask C.

\begin{tabular}{l|rr} 
System & F1 $_{1}$ macro & Acc. \\
\hline All IND baseline & 0.3041 & 0.8387 \\
All GRP baseline & 0.0762 & 0.1290 \\
All OTH baseline & 0.0208 & 0.0323 \\
\hline SVM $_{\text {character-ngram }}$ & 0.3915 & 0.8065 \\
SVM $_{\text {word-ngram }}$ & 0.3554 & 0.6774 \\
SVM $_{\text {char+ling+emoji }}$ & $\mathbf{0 . 3 9 7 1}$ & 0.8065 \\
SVM $_{\text {char+ling+emoji+entity }}$ & 0.3901 & 0.7742 \\
\hline
\end{tabular}

Table 5: Results on the trial data for subtask C.

sistently noticed that the BERT classifier is highly effective in identifying tweets with words that are negative or offensive in most linguistic contexts. The real challenge is that not all tweets containing negative or potentially insulting words are offensive; there are subtle differences between a negative opinion and an insult towards someone. However, the model cannot distinguish these subtle differences in meaning in the proper cultural or socio-political contexts. Additionally, it is not robust enough to detect swear word variants or atypical spellings common in social media.

\subsection{Subtask C}

Table 4 shows our best result for subtask $\mathrm{C}$ in comparison to the official baselines. The macro $\mathrm{F}_{1}$ score of the SVM classifier is 0.5243 , which is considerably higher than the baseline and ranks the system 27th out of 65 submissions. The confusion matrix in Figure 2 indicates that our classi- 


\begin{tabular}{l|l|l|l} 
ID & Tweet & Label & Prediction \\
\hline 17 & $\begin{array}{l}\text { @User Obama fed the country shit sandwiches for 8 years. Maybe Jim } \\
\text { just has his addled mind confused about dates and who fed who what.. }\end{array}$ & GRP & IND \\
\hline 22 & @User The Catholic Church is really screwed up. Nothing new here. & GRP & OTH \\
\hline 31 & $\begin{array}{l}\text { @User Yeah thanks to your Nobel Emmy award winning idiot chief flip } \\
\text { flopping on everything from Iran to gun control. }\end{array}$ & IND & GRP \\
\hline
\end{tabular}

Table 6: Misclassified examples from the trial data for subtask C.

fier performed well on identifying the IND class, was effective for the GRP class, but often failed to distinguish the OTH class from the other two classes. This clearly shows that the sparsity of training data for the minority class OTH affects the performance of our classifier negatively.

The performance of the classifier with different features is shown in Table 5. Since there are only 31 instances in the trial set and it is rather imbalanced, we can see that the highest accuracy is reached by classifying all examples as IND, i.e., the all IND baseline. Even though none of the classifiers outperformed the baseline in terms of accuracy, all the classifiers achieved significantly higher macro $F_{1}$ scores, which shows that they are better at identifying the other two classes. After adding linguistic and emoji features, the character $n$-gram model showed a slight improvement in macro $F_{1}$ score and achieved the highest accuracy along with the simple character $n$-gram model. But both macro $F_{1}$ and accuracy dropped when entity information was added.

Table 6 presents examples of misclassified tweets in the trial set. In example 17, two persons are mentioned, "Obama" and "Jim', and both of them are insulted, however not as a group but individually. The classifier labeled this example as IND. In example 22, the classifier is misguided by the word 'Church' and wrongly classifies it as OTH. Example 31 is similar to example 17. Here, there are two potential targets, 'Nobel Emmy award winning idiot' and 'Iran' that could trigger the group sense, which significantly affects the classifier's judgment.

The errors analysis indicates that the classifier has the ability to distinguish individual and group targets, but it fails to capture the relation between different entities and sometimes misidentifies the target category of offensive language.

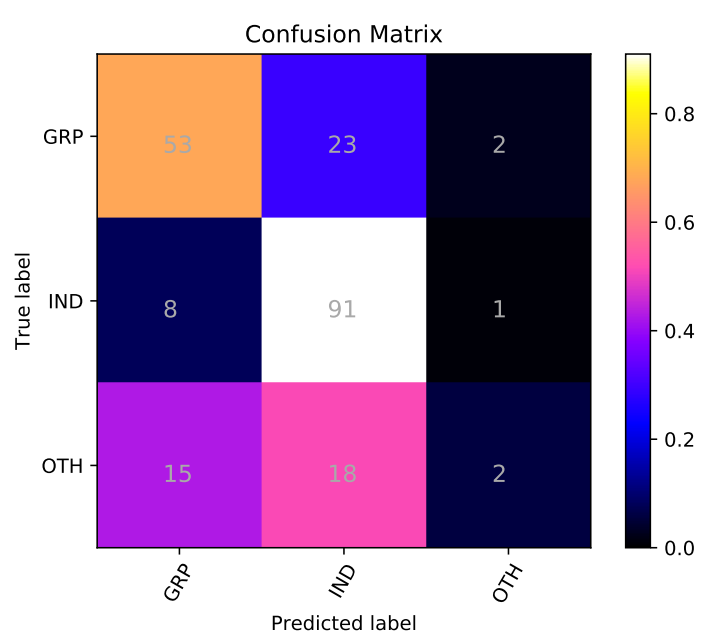

Figure 2: Confusion matrix for the SVM classifier for subtask $\mathrm{C}$.

\section{Conclusion}

In this study, we report our systems for OffensEval subtasks A and C. In subtask A, we trained a neural network based classifier by fine-tuning the pretrained BERT $\mathrm{T}_{\text {base }}$ model to detect offensive tweets. In subtask $C$, we used a linear SVM with character $n$-gram features to identify the target of hate speech.

The evaluation results indicate that our system is capable of detecting offensive language robustly, and it has a good chance of identifying the target. However, there is room for improvement. In the future, in order to capture subtle meaning and overcome the data sparsity, we plan to take syntactic and semantic features into consideration and investigate the combination of selected surface features and pre-trained word embeddings.

\section{References}

Ignacio Arroyo-Fernández, Dominic Forest, JuanManuel Torres-Moreno, Mauricio Carrasco-Ruiz, Thomas Legeleux, and Karen Joannette. 2018. Cyberbullying detection task: The EBSI-LIA-UNAM system (ELU) at COLING'18 TRAC-1. In Proceed- 
ings of the First Workshop on Trolling, Aggression and Cyberbullying (TRAC-2018), pages 140-149.

Pinkesh Badjatiya, Shashank Gupta, Manish Gupta, and Vasudeva Varma. 2017. Deep learning for hate speech detection in tweets. In Proceedings of the 26th International Conference on World Wide Web Companion, pages 759-760, Perth, Australia.

Pete Burnap and Matthew L Williams. 2015. Cyber hate speech on twitter: An application of machine classification and statistical modeling for policy and decision making. Policy \& Internet, 7(2):223-242.

Thomas Davidson, Dana Warmsley, Michael Macy, and Ingmar Weber. 2017. Automated hate speech detection and the problem of offensive language. In Proceedings of ICWSM.

Jacob Devlin, Ming-Wei Chang, Kenton Lee, and Kristina Toutanova. 2018. BERT: Pre-training of deep bidirectional transformers for language understanding. arXiv preprint arXiv:1810.04805.

Karthik Dinakar, Roi Reichart, and Henry Lieberman. 2011. Modeling the detection of textual cyberbullying. In The Social Mobile Web, pages 11-17.

Nemanja Djuric, Jing Zhou, Robin Morris, Mihajlo Grbovic, Vladan Radosavljevic, and Narayan Bhamidipati. 2015. Hate speech detection with comment embeddings. In Proceedings of the 24th International Conference on World Wide Web Companion, pages 29-30.

Antigoni Maria Founta, Constantinos Djouvas, Despoina Chatzakou, Ilias Leontiadis, Jeremy Blackburn, Gianluca Stringhini, Athena Vakali, Michael Sirivianos, and Nicolas Kourtellis. 2018. Large scale crowdsourcing and characterization of twitter abusive behavior. In Twelfth International AAAI Conference on Web and Social Media.

Efthymios Kouloumpis, Theresa Wilson, and Johanna Moore. 2011. Twitter sentiment analysis: The good the bad and the OMG! In Fifth International AAAI Conference on Weblogs and Social Media.

Sandra Kübler, Can Liu, and Zeeshan Ali Sayyed. 2018. To use or not to use: Feature selection for sentiment analysis of highly imbalanced data. Natural Language Engineering, 24(1):3-37.

Ritesh Kumar, Atul Kr Ojha, Shervin Malmasi, and Marcos Zampieri. 2018. Benchmarking aggression identification in social media. In Proceedings of the First Workshop on Trolling, Aggression and Cyberbullying (TRAC-2018), pages 1-11.

Irene Kwok and Yuzhou Wang. 2013. Locate the hate: Detecting tweets against blacks. In Twenty-Seventh AAAI Conference on Artificial Intelligence.

Shervin Malmasi and Marcos Zampieri. 2018. Challenges in discriminating profanity from hate speech. Journal of Experimental \& Theoretical Artificial Intelligence, 30:1-16.
Tomas Mikolov, Ilya Sutskever, Kai Chen, Greg S Corrado, and Jeff Dean. 2013. Distributed representations of words and phrases and their compositionality. In Advances in Neural Information Processing Systems, pages 3111-3119.

Chikashi Nobata, Joel Tetreault, Achint Thomas, Yashar Mehdad, and Yi Chang. 2016. Abusive language detection in online user content. In Proceedings of the 25th International Conference on World Wide Web, pages 145-153.

Petra Kralj Novak, Jasmina Smailović, Borut Sluban, and Igor Mozetič. 2015. Sentiment of emojis. PloS One, 10(12).

Adam Paszke, Sam Gross, Soumith Chintala, Gregory Chanan, Edward Yang, Zachary DeVito, Zeming Lin, Alban Desmaison, Luca Antiga, and Adam Lerer. 2017. Automatic differentiation in PyTorch. In NIPS-W

Fabian Pedregosa, Gaël Varoquaux, Alexandre Gramfort, Vincent Michel, Bertrand Thirion, Olivier Grisel, Mathieu Blondel, Peter Prettenhofer, Ron Weiss, Vincent Dubourg, et al. 2011. Scikit-learn: Machine learning in Python. Journal of Machine Learning Research, 12:2825-2830.

Jeffrey Pennington, Richard Socher, and Christopher Manning. 2014. GloVe: Global vectors for word representation. In Proceedings of the 2014 Conference on Empirical Methods in Natural Language Processing (EMNLP), pages 1532-1543.

Matthew Peters, Mark Neumann, Mohit Iyyer, Matt Gardner, Christopher Clark, Kenton Lee, and Luke Zettlemoyer. 2018. Deep contextualized word representations. In Proceedings of the 2018 Conference of the North American Chapter of the Association for Computational Linguistics: Human Language Technologies, pages 2227-2237.

Alec Radford, Karthik Narasimhan, Tim Salimans, and Ilya Sutskever. 2018. Improving language understanding by generative pre-training. URL: https://s3-us-west-2.amazonaws. com/openai-assets/research-covers/ languageunsupervised/

languageunderstandingpaper.pdf.

Anna Schmidt and Michael Wiegand. 2017. A survey on hate speech detection using natural language processing. In Proceedings of the Fifth International Workshop on Natural Language Processing for Social Media, pages 1-10, Valencia, Spain.

Mohammed Shiha and Serkan Ayvaz. 2017. The effects of emoji in sentiment analysis. International Journal of Computer and Electrical Engineering (IJCEE.), 9(1):360-369.

Yonghui Wu, Mike Schuster, Zhifeng Chen, Quoc V. Le, Mohammad Norouzi, Wolfgang Macherey, Maxim Krikun, Yuan Cao, Qin Gao, Klaus Macherey, Jeff Klingner, Apurva Shah, Melvin 
Johnson, Xiaobing Liu, Lukasz Kaiser, Stephan Gouws, Yoshikiyo Kato, Taku Kudo, Hideto Kazawa, Keith Stevens, George Kurian, Nishant Patil, Wei Wang, Cliff Young, Jason Smith, Jason Riesa, Alex Rudnick, Oriol Vinyals, Greg Corrado, Macduff Hughes, and Jeffrey Dean. 2016. Google's neural machine translation system: Bridging the gap between human and machine translation. arXiv preprint arXiv:1609.08144.

Marcos Zampieri, Shervin Malmasi, Preslav Nakov, Sara Rosenthal, Noura Farra, and Ritesh Kumar. 2019a. Predicting the Type and Target of Offensive Posts in Social Media. In Proceedings of NAACL.

Marcos Zampieri, Shervin Malmasi, Preslav Nakov, Sara Rosenthal, Noura Farra, and Ritesh Kumar. 2019b. SemEval-2019 Task 6: Identifying and Categorizing Offensive Language in Social Media (OffensEval). In Proceedings of The 13th International Workshop on Semantic Evaluation (SemEval). 